Taraz State University after M.Kh.Dulaty,

Taraz, Kazakhstan

p-ISSN: 2308-4944 (print)

e-ISSN: 2409-0085 (online)

Year: 2017

Issue: 05

Volume: 49

Published: 1.05.2017 http://T-Science.org

SECTION 1. Theoretical research in mathematics.

\title{
ABOUT ONE APPROACH OF SOLVING THE PROBLEM OF OPTICAL SENSING
}

Abstract: The paper considers one inverse problem of hydro-optics. The importance of these tasks taking into account multiple scattering increases with the necessity of interpretation of optical observations in probing. The aqueous medium in which the radiation propagates is supposed layered-homogeneous. The linkage between the characteristics and parameters of the test medium contributes to the formulation and successful solution of inverse problems. After that by direct simulation of photon trajectories on a computer the problem of determining the scattering coefficient is solved using the Monte Carlo method.

Key words: Monte-Carlo methods, integral equations, optics of the water environment.

Language: English

Citation: Seytkerim AS (2017) ABOUT ONE APPROACH OF SOLVING THE PROBLEM OF OPTICAL SENSING. ISJ Theoretical \& Applied Science, 05 (49): 75-79.

Soi: http://s-o-i.org/1.1/TAS-05-49-14 Doi: crossef https://dx.doi.org/10.15863/TAS.2017.05.49.14

\section{UDC 519.245+519.642}

\section{Introduction}

Description of the task. Let in the aquatic environment $\quad 0<H_{\text {sur. }} \leq z \leq H_{\text {bot }}, \quad(x, y) \in R^{2}$ spreads narrow light beam of constant wavelength, emitted from a point source $\vec{r}_{\text {sou }}=(0,0,0)$, placed in «vacuum» above the surface of the medium, isotropically in a circular conical solid angle $\Omega_{\text {sou. }}=\left[\cos \theta_{\text {sou. }}, 1\right] \times[0, \pi]$, where $\Omega_{\text {sou. }}$ - the angle of the source relative to the unit vector $\vec{n}_{\text {sou. }}=(0,0,1)$ the axis of the source. The density distribution emitted by the source of light quanta is given by function [1]

$$
\psi(\vec{r}, \vec{\omega}, t)=p(\vec{\omega}) \delta\left(\vec{r}-\vec{r}_{\text {sou. }}\right) \delta(t)
$$

where $p(\vec{\omega})=\left\{\begin{array}{c}\left\{2 \pi\left(1-\cos \theta_{\text {sou. }}\right)\right\}^{-1}, \quad \text { if } \vec{\omega} \in \Omega_{\text {sou. }} \\ 0, \quad \text { otherwise. }\end{array}\right.$

The environment is expected to horizontally homogeneous and broken $z$ axis into $n$ sufficiently thin layers with different constant values of scattering coefficient $\sigma_{s_{1}}^{*}, \sigma_{s_{2}}^{*}, \ldots, \sigma_{s_{n}}^{*}$ inside them. The value of the absorption coefficient is considered in all layers of constant and equal $\sigma_{a}^{*}$. The numbering of the layers are from top to bottom.

The bottom $z=H_{b o t}$, with a value of albedo Alprobability of survival, reflects radiation according to Lambert law: the density distribution of the cosine of the angle $\theta_{0}$ between the reflected beam from the bottom surface and the normal to it has the form

$$
p_{0}(\mu)=2 \mu, \quad \mu=\cos \theta_{0} .
$$

Receiver with optical axis along the vector $\vec{n}_{\text {rec. }}=(0,0,1)$, measuring the intensity of scattered radiation environment, combined with the source and perceives only the light, supplied in directions $\vec{\omega}$ such that $\left(\vec{\omega}, \vec{n}_{r e c}\right) \geq \cos \theta_{\text {rec. }}$, that is $\vec{\omega} \in \Omega_{\text {rec. }}=\left[\cos \theta_{\text {rec. }}, 1\right] \times[0, \pi] \quad-$ the angle of the receiver.

Let us consider the temporal distribution of the intensity of the radiation entering the receiver. The total value of the intensity is

$$
I(t)=\int_{\Omega_{r e c .}} \Phi\left(\vec{r}_{r e c .}, \vec{\omega}, t\right) d \vec{\omega}
$$




\begin{tabular}{|c|c|c|c|c|c|c|}
\hline \multirow{4}{*}{ Impact Factor: } & ISRA (India) & $=1.344$ & SIS (USA) & $=0.912$ & \multirow{4}{*}{$\begin{array}{l}\text { ICV (Poland) } \\
\text { PIF (India) } \\
\text { IBI (India) }\end{array}$} & \multirow{4}{*}{$\begin{array}{l}=6.630 \\
=1.940 \\
=4.260\end{array}$} \\
\hline & ISI (Dubai, UAF & $=0.829$ & РИНЦ (Russia) & $=0.234$ & & \\
\hline & GIF (Australia) & $=0.564$ & ESJI (KZ) & $=3.860$ & & \\
\hline & JIF & $=1.500$ & SJIF (Morocco) & $=\mathbf{2 . 0 3 1}$ & & \\
\hline
\end{tabular}

The temporal distribution of $I_{k}=\frac{1}{t_{k}-t_{k-1}} \int_{t_{k-1}}^{t_{k}} I(t) d t$ this intensity in intervals $\left[t_{k-1}, t_{k}\right]$, where $t_{k}$ - the nodes of the breaking length of time $[0, T], k=1, \ldots, m$, it is possible to measure experimentally.

Statement of the problem. Knowing the different time intervals, find the value of the scattering coefficient of the medium in layers $1, \ldots, n$, at a known and fixed value of absorption coefficient in all layers [2].

The solution of the task. $I_{k}$ it is a function of the value $\sigma=\sigma_{s}+\sigma_{a}: \quad I_{k}=I_{k}(\sigma)$. It's known $[3,4]$, that measured intensity values $I_{1}, I_{2}, \ldots, I_{m}$ in $m$

$$
I_{k}(\sigma)=\sum_{j=0}^{\infty} \int_{X} \ldots \int_{X} \psi\left(\vec{x}_{0}\right) \prod_{i=0}^{j-1} k\left(\vec{x}_{i}, \vec{x}_{i+1}, \sigma\right) \varphi_{k}\left(\vec{x}_{j}, \sigma\right) d \vec{x}_{0} \ldots d \vec{x}_{j-1} d \vec{x}_{j}
$$

where

$$
\begin{aligned}
k\left(\vec{x}_{i}, \vec{x}_{i+1}, \sigma\right)=\frac{q\left(\vec{r}_{i}\right) g(\mu) \sigma \exp \left(-\sigma\left|\vec{r}_{i+1}-\vec{r}_{i}\right|\right)}{2 \pi\left|\vec{r}_{i+1}-\vec{r}_{i}\right|^{2}} \delta\left(\vec{\omega}_{i+1}-\frac{\vec{r}_{i+1}-\vec{r}_{i}}{\left|\vec{r}_{i+1}-\vec{r}_{i}\right|}\right) \times \\
\times \delta\left(t_{i+1}-t_{i}-\frac{\left|\vec{r}_{i+1}-\vec{r}_{i}\right|}{v}\right)
\end{aligned}
$$

$\vec{r}_{i}$ - the radius vector of point $i$-th collision,

$\vec{\omega}_{i}-$ a unit vector in the direction of the photon before $i$-th collision, $t_{i}-$ time $i$-th collision, measured from the moment of penetration of a photon in the environment,

$$
\begin{aligned}
& \vec{x}_{i}=\left(\vec{r}_{i}, \vec{\omega}_{i}, t_{i}\right), \\
& \mu=\left(\vec{\omega}_{i}, \vec{\omega}_{i+1}\right), \\
& \varphi_{k}\left(\vec{x}_{j}, \sigma\right)=\sigma \exp \left(-\sigma\left|\vec{r}_{\text {sur. }}-\vec{r}_{j}\right|\right) F_{1}, \\
& F_{1}=\frac{q\left(\vec{r}_{j}\right) g\left(\mu_{1}\right)}{2 \pi\left|\vec{r}_{\text {sur. }}-\vec{r}_{j}\right|^{2}} \Delta_{\Omega_{\text {rec. }}}\left(\vec{\omega}_{\text {rec. }}\right) \Delta_{k}\left(t^{*}\right)\left\{1-Y\left(\vec{\omega}_{\text {sur. }}\right)\right\}, \\
& \vec{r}_{\text {sur }}-\text { the radius of the intersection vector of the } \\
& \text { photon with the medium surface when returning to } \\
& \text { the } \quad \vec{r}_{j}, \\
& \vec{\omega}_{\text {sur. }}=\left(\vec{r}_{\text {sur. }}-\vec{r}_{j}\right) /\left|\vec{r}_{\text {sur. }}-\vec{r}_{j}\right|, \\
& \mu_{1}=\left(\vec{\varpi}_{j}, \vec{\varpi}_{\text {sur }}\right)-\text { cosine of the angle between }
\end{aligned}
$$

the $\vec{\varpi}_{j}$ and $\vec{\omega}_{\text {sur. }}$,

$q\left(\vec{r}_{j}\right), q\left(\vec{r}_{i}\right)$ probability of survival,

$$
\vec{\omega}_{\text {rec. }}=\left(\vec{r}_{\text {rec. }}-\vec{r}_{\text {sur. }}\right) /\left|\vec{r}_{\text {rec. }}-\vec{r}_{\text {sur. }}\right|,
$$$$
\Delta_{\Omega_{r e c}}\left(\vec{\omega}_{r e c .}\right)=\left\{\begin{array}{ll}
1, & \text { if } \\
0, & \text { otherwise }
\end{array} \vec{\omega}_{\text {rec. }} \in \Omega_{\text {rec. }},-\right. \text { the }
$$

$t^{*}=t_{0}+t_{j}+t_{\text {sur. }}+t_{\text {rec. }}-$ the total time of a photon in transit from source to receiver,

$$
t_{0}=H_{\text {sur. }} / \mu_{0} c \text {, where } \vec{\omega}_{0}=\left(\mu_{0}, \beta_{0}\right)-\text { the }
$$
random vector of the direction of departure of a photon from a source with a given distribution density $p(\vec{\omega}), c$ - the speed of light in vacuum. $t_{j}-$ the time of the collision at the point $\vec{r}_{j}$, starting from the contact with the medium, $t_{\text {sur }}=\left|\vec{r}_{\text {sur. }}-\vec{r}_{j}\right| / v-$ time to reach the surface of the medium photon at time $t_{j}, v$ - the speed of light in water, $t_{\text {rec. }}=\left|\vec{r}_{\text {rec }}-\vec{r}_{\text {sur. }}\right| / c-$ the time of reaching the photon receiver after it's left of the environment, $\Delta_{k}\left(t^{*}\right)= \begin{cases}1, & \text { if } t^{*} \in\left[t_{k-1}, t_{k}\right], \quad-\text { indicator } k \text {-th } \\ 0, & \text { otherwise }\end{cases}$ time interval, $Y\left(\vec{\omega}_{\text {sur. }}\right)-$ frenel reflection coefficient at point $\vec{r}_{\text {sur. }}$.

To find $\sigma_{s_{1}}^{*}, \sigma_{s_{2}}^{*}, \ldots, \sigma_{s_{n}}^{*}$ in the work [6] the proposed recurrence system of linear equations

$$
\sum_{j=1}^{m} q_{i j}^{(p)} \Delta \sigma_{s_{j}}^{(p+1)}=d_{i}^{(p)}
$$

where

angle indicator of solubility of the receiver, 


\begin{tabular}{|c|c|c|c|c|c|c|}
\hline Impact Factor: & $\begin{array}{l}\text { ISRA (India) } \\
\text { ISI (Dubai, UAE } \\
\text { GIF (Australia) } \\
\text { JIF }\end{array}$ & $\begin{array}{l}=1.344 \\
=0.829 \\
=0.564 \\
=1.500\end{array}$ & $\begin{array}{l}\text { SIS (USA) } \\
\text { PИНЦ (Russia) } \\
\text { ESJI (KZ) } \\
\text { SJIF (Morocco) }\end{array}$ & $\begin{array}{l}=0.912 \\
=0.234 \\
=3.860 \\
=\mathbf{2 . 0 3 1}\end{array}$ & $\begin{array}{l}\text { ICV (Poland) } \\
\text { PIF (India) } \\
\text { IBI (India) }\end{array}$ & $\begin{array}{l}=6.630 \\
=1.940 \\
=4.260\end{array}$ \\
\hline
\end{tabular}

$$
\begin{gathered}
i=1, \ldots, n, \quad q_{i j}^{(p)}=\sum_{k=1}^{m} a_{k i}^{(p)} a_{k j}^{(p)}, \quad d_{i}^{(p)}=\sum_{k=1}^{m} a_{k i}^{(p)} b_{k}^{(p)}, a_{k i}^{(p)}=\left.\frac{\partial I_{k}(\vec{\sigma})}{\partial \sigma_{s_{i}}}\right|_{\vec{\sigma}=\vec{\sigma}^{(p)}}, \quad a_{k j}^{(p)}=\left.\frac{\partial I_{k}(\vec{\sigma})}{\partial \sigma_{s_{j}}}\right|_{\vec{\sigma}=\vec{\sigma}^{(p)}}, \\
b_{k}^{(p)}=I_{k}^{*}-I_{k}\left(\vec{\sigma}^{(p)}\right), \quad \Delta \sigma_{s_{j}}^{(p+1)}=\sigma_{s_{j}}^{(p+1)}-\sigma_{s_{j}}^{(p)},
\end{gathered}
$$

$I_{k}^{*}-$ the values of the intensity of scattered

radiation observed by the receiver in time intervals

$$
k=1, \ldots, m, \vec{\sigma}=\vec{\sigma}_{s}+\vec{\sigma}_{a}^{*}, \quad \vec{\sigma}_{s}=\left(\sigma_{s_{1}}, \ldots, \sigma_{s_{n}}\right) . \vec{\sigma}_{a}^{*}=\left(\sigma_{a}^{*}, \ldots, \sigma_{a}^{*}\right),
$$

$\sigma_{s_{1}}^{(p)}, \ldots, \sigma_{s_{n}}^{(p)}-p$-th approximation to the expected

values.

In research we will use the following

well-known formulas the approximate formulas[7]:

$$
\begin{gathered}
I_{k}\left(\vec{\sigma}^{(p)}\right)=\frac{1}{N} \sum_{l=1}^{N} \xi_{l}^{(p)}(k), \quad a_{k h}^{(p)}=\frac{1}{N} \sum_{l=1}^{N} \eta_{l}^{(p)}(k, h), \text { where } \\
\xi_{l}^{(p)}(k)=\sum_{j=0}^{\gamma(l)} \varphi_{k}\left(x_{j}^{(l)}, \vec{\sigma}^{(p)}\right), \quad \eta_{l}^{(p)}(k, h)=\sum_{j=0}^{\gamma(l)} \varphi_{k}\left(x_{j}^{(l)}, \vec{\sigma}^{(p)}\right) w_{j}^{(l)}\left(\sigma_{h}^{(p)}\right), \\
w_{j}^{(l)}\left(\sigma_{h}^{(p)}\right)=\frac{M_{h}^{(j+1)}}{\sigma_{h}^{(p)}}-\sum_{i=0}^{j-1} l_{h}^{(i, i+1)}-l_{h}^{(j)},
\end{gathered}
$$

$\vec{r}_{0}^{(l)}, \vec{r}_{1}^{(l)}, \ldots, \vec{r}_{j}^{(l)}-$ the collision point $l$-th the simulated trajectory.

$l_{h}^{(i, i+1)}, l_{h}^{(j)}-$ the length of those parts of the segments $\left|\vec{r}_{i+1}^{(l)}-\vec{r}_{i}^{(l)}\right|$ и $\left|\vec{r}_{\text {sur }}^{(l)}-\vec{r}_{j}^{(l)}\right|$, which accounted for $h$-th layer,

$M_{h}^{(j+1)}$ the number of points from the set $\vec{r}_{0}^{(l)}, \vec{r}_{1}^{(l)}, \ldots, \vec{r}_{j}^{(l)}$, caught in $h$-th layer, $\gamma(l)-$ random number collision, which ends up with $l$-th trajectory [8].

The objective of the evaluation values $I_{k}^{(p)}=I_{k}\left(\vec{\sigma}^{(p)}\right)$ and their derivatives $a_{k i}^{(p)}$, $p=0,1,2, \ldots$ shall be determined by direct simulation of the photon trajectories on a computer, as a homogeneous Markov chain of random points on collision $x_{0}, x_{1}, \ldots, x_{n}, \ldots$ of a photon with the elements of the water environment. Distribution of the initial state of the Markov chain is specified by the function $\psi(x)$, physical distribution density of the emitted light quanta. The transition density of the Markov chain from one state to another state is set by the physical density of transitions, the given function (1). The solution in this article the inverse problem, in fact, it is the solution of a series of direct problems. Namely, at the beginning of the true values $\left\{\sigma_{s_{i}}^{*}\right\}_{i=1, \ldots n}$ estimated intensity values $\left\{I_{k}^{*}\right\}_{k=1, \ldots, m}$ and its derivatives $\left\{a_{k i}^{*}\right\}_{k=1, \ldots, m, i=1, . . n}$ and

they are accepted as "measured" by the device, in the General case getting a faux, normally distributed random error $\left\{\varsigma_{k}\right\}_{k=1, \ldots m}$, owing to the inaccuracy of physical experiments. Next selected prognostic values of the unknown factor $\left\{\sigma_{s_{i}}^{(0)}\right\}_{i=1, \ldots n}$ in layers, and for them again solved the direct problem in the calculation of $\left\{I_{k}^{(0)}\right\}_{k=1, \ldots, m}$ and $\left\{a_{k i}^{(0)}\right\}_{k=1, \ldots, m, i=1, . ., n}$. According to the latest system of equations is solved (2) relatively $\left\{\sigma_{s_{i}}^{(1)}\right\}_{i=1, \ldots n}$ and etc.

Practical solution of the problem by Monte Carlo. To study the effectiveness of various modifications of the process simulation of radiation transfer with strongly anisotropic scattering can use the following process model. The particle moves from point $x=0$ along the axis $x$ random runs, distributed with density $e^{-x}(x>0)$. At the end of the path there is a collision, in which a particle can be absorbed with a probability $1-q$; otherwise, she's moving on. At the point $x=H$ occurs sortie, i.e. torn off trajectory. Required to calculate the probability that the particle will leave, having experienced at least one collision. The transition density for the chain of collisions is determined by the formula [9]:

$$
k\left(x^{\prime}, x\right)=q e^{-\left(x-x^{\prime}\right)}, x^{\prime} \leq x \leq H
$$




\begin{tabular}{|c|c|c|c|c|c|c|}
\hline Impact Factor: & $\begin{array}{l}\text { ISRA (India) } \\
\text { ISI (Dubai, UAE } \\
\text { GIF (Australia) } \\
\text { JIF }\end{array}$ & $\begin{array}{l}=1.344 \\
=0.829 \\
=0.564 \\
=1.500\end{array}$ & $\begin{array}{l}\text { SIS (USA) } \\
\text { PИНЦ (Russia) } \\
\text { ESJI (KZ) } \\
\text { SJIF (Morocco) }\end{array}$ & $\begin{array}{l}=0.912 \\
=0.234 \\
=3.860 \\
=\mathbf{2 . 0 3 1}\end{array}$ & $\begin{array}{l}\text { ICV (Poland) } \\
\text { PIF (India) } \\
\text { IBI (India) }\end{array}$ & $\begin{array}{l}=6.630 \\
=1.940 \\
=4.260\end{array}$ \\
\hline
\end{tabular}

The density of the primary collision $f(x)=e^{-x}(0 \leq x \leq H)$. Therefore, the total density of the collision $\varphi(x)$ satisfies the equation

$$
\varphi(x)=q \int_{0}^{\pi} e^{-\left(x-x^{\prime}\right)} \varphi\left(x^{\prime}\right) d x^{\prime}+e^{-x}
$$

It is easy to check that here $\|K\| \prec q$. It is known that in this case the required probability $P=\varphi(H)$. Thus, it is required to compute the solution of the integral equation at a single point $x=H$. To do this, you can use a local assessment, putting $h(x)=k(x, H)=q e^{-(H-x)}$, i.e. $P=M \xi$, where $\xi=\sum_{n=0}^{N} h\left(x_{n}\right)=\sum_{n=0}^{N} q e^{-\left(H-x_{n}\right)}$, if implemented direct modeling a chain collision [10]. Direct substitution into the equation can see that here

$$
\varphi(x)=e^{-(1-q) x}, 0 \leq x \leq H
$$

It is easy to obtain the value of the required probability

$$
P=\varphi(H)=(\varphi, h)=e^{-(1-q) H}\left(1-e^{-q H}\right) .
$$

The adjoint equation in this case has the form

$$
\varphi^{*}(x)=q \int_{x}^{H} e^{-\left(x^{\prime}-x\right)} \varphi^{*}\left(x^{\prime}\right) d x^{\prime}+q e^{-(H-x)} .
$$

Him meets function $\varphi^{*}(x)=q e^{-(1-q)(H-x)}$. The variance of the random evaluation $\xi$ is determined by the equality

$$
M \xi^{2}=\left(\varphi, h\left(2 \varphi^{*}-h\right)\right)=q^{2} \frac{1+2 q}{1+q} e^{-(1-q) H}-2 q^{2} e^{-H}+\frac{q^{2} e^{-2 H}}{1+q}
$$

Consider now the evaluation on acquisitions

$$
\eta=\frac{h\left(x_{N}\right)}{g\left(x_{N}\right)}=\frac{q e^{-\left(H-x_{N}\right)}}{1-q+q e^{-\left(H-x_{N}\right)}} .
$$

For her

$$
M \eta^{2}=\left(\varphi, \frac{h^{2}}{g}\right)=q^{2} e^{-(1-q) H} \int_{0}^{H} \frac{e^{-(1+q) x}}{1-q+q e^{-x}} d x .
$$

In this case, we can consider a binary evaluation $\zeta$, which is equal to 1 , if the particle flew, and equal to 0 otherwise. It is obvious that
$M \zeta^{2}=M \zeta=P=e^{-(1-q) H}\left(1-e^{-q H}\right)$. To compare variance estimates for a large $H$ it is enough to consider the corresponding coefficients of the functions $e^{-(1-q) H}$, i.e. value

$$
C_{\xi}=q^{2} \frac{1+2 q}{1+q}, C_{\eta}=q^{2} \int_{0}^{\infty} \frac{e^{-(1+q) x}}{1-q+q e^{-x}} d x, C_{\zeta}=1
$$

Values of $C_{\xi}$ and $C_{\eta}$ given in the table 1.

The values of the coefficients $C_{\xi}$ and $C_{\eta}$.

Table 1

\begin{tabular}{|l|l|l|l|l|l|l|l|l|l|l|}
\hline$q$ & 0,1 & 0,2 & 0,3 & 0,4 & 0,5 & 0,6 & 0,7 & 0,8 & 0,9 & 1 \\
\hline$C_{\xi}$ & 0,0101 & 0,047 & 0,111 & 0,21 & 0,33 & 0,45 & 0,62 & 0,93 & 1,19 & 1,5 \\
\hline$C_{\eta}$ & 0,0096 & 0,037 & 0,080 & 0,14 & 0,21 & 0,31 & 0,42 & 0,55 & 0,72 & 1 \\
\hline
\end{tabular}

\section{Conclusion.}

Thus, can be seen, evaluation on absorption multiple is more accurate than the other evaluation. This is due to the fact, in this case, the probability is calculated from the absorbance of one species sortie. In the transition to the real problems of the relationship between $D \xi$ and $D \eta$ changes in the valuation advantage $\xi$ because of the possibility of more changes $g(x)$. Also in the works, knowing the measured values of intensity in different time intervals, found the value of the scattering coefficient of the medium in layers at a known and fixed value of absorption coefficient. 


\begin{tabular}{l|lrl|l|ll} 
& ISRA (India) & $=\mathbf{1 . 3 4 4}$ & SIS (USA) & $=\mathbf{0 . 9 1 2}$ & ICV (Poland) & $=\mathbf{6 . 6 3 0}$ \\
Impact Factor: & ISI (Dubai, UAE) $=\mathbf{0 . 8 2 9}$ & PUHIL (Russia) $=\mathbf{0 . 2 3 4}$ & PIF (India) & $=\mathbf{1 . 9 4 0}$ \\
& GIF (Australia) & $\mathbf{0 . 5 6 4}$ & ESJI (KZ) & $=\mathbf{3 . 8 6 0}$ & IBI (India) & $\mathbf{= 4 . 2 6 0}$ \\
& JIF & $\mathbf{1 . 5 0 0}$ & SJIF (Morocco) & $=\mathbf{2 . 0 3 1}$ & & \\
\hline
\end{tabular}

\section{References:}

1. Antyufeev VS, Nazaraliev MA (1998) Obratnye zadachi atmosfernoi optiki. - Novosibirsk.

2. Nazaraliev MA (1990) Statisticheskoe modelirovanie radiacionyh processov $\mathrm{V}$ atmosfere. -Novosibirsk: Nauka.

3. Mikhailov GA (1974) Nekotorye voprosy teorii metodov Monte-Carlo. - Novosibirsk: Nauka.

4. Ermakov SM, Mikhaylov GA (1982) Statisticheskoe modelirovanie.-Moscow: Nauka.

5. Romanovski VI (1949) Diskretnye chepi Markova. - M.:Gostehizdat.
6. Dynkin EB (1963) Markovskye processy. -M .: Fizmatgiz.

7. Bartlett MS (1958) Vvedenye v teoryu sluchaynyh processov. M:. IL.

8. Ermakov SM, Mikhailov GA (1976) Kurs statisticheskogo modelirovanya. -M .: Nauka.

9. Sobol IM (1968) Metod Monte-Carlo. -M .: Nauka.

10. Ermakov SM (1975) Metod Monte-Carlo I smejnye voprosy. - M.: Nauka. 